\title{
Noncommutative associative superproduct for general supersymplectic forms
}

\author{
A. De Castro, ${ }^{a}$ I. Martín, ${ }^{a}$ L. Quevedo ${ }^{a}$ and A. Restuccia ${ }^{a b}$ \\ a Departamento de Física, Universidad Simón Bolívar, \\ Ap. 89000, Caracas 1080-A, Venezuela \\ ${ }^{b}$ Max Planck Institut für Gravitationsphysik, Albert Einstein Institut, \\ Am Mühlenberg 1, 14476 Golm, Germany \\ E-mail: adcastro@usb.ve, isbeliam@usb.ve, Iquevedo@usb.ve, arestu@usb.ve
}

\begin{abstract}
We define a noncommutative and nonanticommutative associative product for general supersymplectic forms, allowing the explicit treatment of non(anti)commutative field theories from general nonconstant string backgrounds like a graviphoton field. We propose a generalization of deformation quantization à la Fedosov to superspace, which considers noncommutativity in the tangent bundle instead of base space, by defining the Weyl super product of elements of Weyl super algebra bundles. Super Poincaré symmetry is not broken and chirality seems not to be compromised in our formulation. We show that, for a particular case, the projection of the Weyl super product to the base space gives rise the Moyal product for non(anti)commutative theories.
\end{abstract}

KEYWords: Superspaces, Non-Commutative Geometry. 


\section{Contents}

1. Introduction 1

2. Weyl algebra bundle 3

3. Weyl superalgebra bundles 6

3.1 Symplectic supermanifolds 6

3.2 Weyl superalgebra bundle through a new associative superproduct 9

4. Superspace deformations 12

4.1 Abelian subalgebra and deformations 12

5. Nonanticommutative $Q$-deformations from Weyl superproduct 14

A. Conventions 16

\section{Introduction}

During the last string theory revolutions we have learned that an undeniable relationship between strings and noncommutative field theories exists. As exemplified by the study of $D$-branes in presence of a finite constant $B$-field [1], 2], it seems that geometry can be noncommutatively deformed whenever stringy effects are relevant. In particular cases like that of strings in a constant graviphoton background, the deformation produced lies in the Graßmann sector of superspace whose undeformed coordinates are characterised by anticommutators, giving raise to what has been called non(anti)commutative field theories [3].

Enormous effort has been made understanding both noncommutative and non(anti)commutative field theories and their relation to string theory [⿴囗大 -11]. Most studies introduce a Moyal product to deform the (super)field algebra of known theories, as this is precisely the kind of geometry that results from string constant backgrounds. The resulting theories usually break Lorentz symmetry and, in the non(anti)commutative case, also either supersymmetry or chirality. The Moyal product construction is very satisfactory when dealing with deformations of Euclidean (super)spaces, but breaks down in curved geometry where it should be replaced by a more general object like the Kontsevich product. As an example, the deformed geometry of $D$-brane worldvolumes in curved backgrounds is given to third order in the deformation parameter precisely by such product [12], which unfortunately can only be constructed order by order in a series expansion. On the other hand, ordinary Moyal product-noncommutative field theories suffer from bad causal behaviour when time is treated as a noncommuting coordinate. 
More recently, in view of the limitations inherent to such methods, an alternative noncommutative deformation of gauge theories based on the notion of the Weyl bundle has been proposed [13, 9]. In these works, authors use the powerful machinery developed by Fedosov [14, 15] which consists in a Weyl deformation of a symplectic tangent space, where the symplectic structure plays the role of the deformation parameter. The advantage of using this method is twofold: it allows the description of physical situations where the background field is not constant, and in addition spacetime global symmetries remain unbroken by the deformation itself since it affects the tangent and not the base space. This approach to deformation quantization has been used extensively to study string theory D-branes in non-constant B-fields and more general curved backgrounds [13], supermembrane models [9], the fuzzy sphere [16], the canonical formulation of noncommuative gauge theories [17], and BRST symmetry in quantum field theories [18].

One may naturally ask if its possible to generalize Fedosov's ideas to describe more general physical setups like non(anti)commutative field theories associated to non-constant graviphoton backgrounds. At first sight one could consider an atlas where the problem may be stated in terms of constant fields on each open set, as the Darboux theorem is only valid in the neighbourhood of a point and not globally, but we must then consider highly nontrivial matching conditions on the overlapping of the sets. It is therfore more convenient to directly develop a non(anti)commutative product for general fields extending the known one for constant fields. Also, from a quantum field theoretical point of view, constructing a mechanism that deforms superspace but preserves super Poincaré symmetry (or its Euclidean counterpart) is relevant in its own right. Our work has precisely this goal.

The deformation quantization of certain Poisson brackets in the context of Batchelor supermanifolds using Fedosov's approach was first done by M. Bordemann, [20, 19]. In the present paper we are mostly interested in field theories involving fermionic fields, as we explicitly discuss in section 5. Although the Batchelor approach is an interesting one from a mathematical and physical point of view it does not allow the introduction of fermionic fields, it is for this reason that the Batchelor definition of supermanifolds is not followed in supersymmetric field theory.

In this paper we extend Fedosov's deformation quantization to $\mathcal{N}=1$ superspace. We take a symplectic supermanifold and ordinary superspace as the tangent and base space respectively, and propose a new associative Weyl product which is non(anti)commutative, meaning it is noncommutative for even objects that naturally fulfil commutation relations, and nonanticommutative for odd objects naturally satisfying anticommutation relations. Such product endows the space of superfields with a superalgebra structure. A key feature of our formulation is the preservation of super Poincaré symmetry, a fact derived from a deformation operator acting exclusively on tangent superspace variables. The super Poincaré algebra acting on base superspace is therefore totally transparent to such deformation. We prove that in certain cases, the projection of the Weyl (super)product on the base supermanifold, results in the nonanticommutative product associated to nilpotent Q- and D-deformations such as those studied in [4, 6-8]. And further explore more general situations where the nonanticommuting parameter is actually a function of the base supermanifold variables. This last subject is not even formulated in the literature as a 
supersymmetric extension using Kontsevich methods. 21].

\section{Weyl algebra bundle}

In this section we review Weyl algebra bundle theory basics [15]. We start with a symplectic manifold $(M, \omega)$ of dimension $2 n$, where the two-form $\omega$ defines a symplectic structure on each tangent space $T_{x}(M), x \in M$.

The formal Weyl algebra $W_{x}$ is an associative algebra of formal series over $\mathbb{C}$ which stems from the symplectic space $T_{x}(M)$. Its elements are defined by

$$
a(y, \hbar)=\sum_{k,|\alpha| \geq 0} \hbar^{k} a_{k, \alpha} y^{\alpha}
$$

with $\hbar$ being the formal parameter, $y \in T_{x}(M)$ with coordinates $\left(y^{1}, \ldots, y^{2 n}\right)$, and $\alpha=$ $\alpha_{1}, \ldots, \alpha_{2 n}$ a multi-index such that $y^{\alpha}=\left(y^{1}\right)^{\alpha_{1}} \cdots\left(y^{2 n}\right)^{\alpha_{2 n}}$. It is possible to introduce an ordering prescription for the terms if we define the degree of the variables to be $\operatorname{deg} y^{i}=$ $1, \operatorname{deg} \hbar=2$. The terms in the series can then be arranged by increasing degrees $2 k+|\alpha|$. A more friendly notation is

$$
a(y, \hbar)=\sum_{k, p \geq 0} \hbar^{k} a_{k, \mu_{1} \ldots \mu_{p}} y^{\mu_{1}} \ldots y^{\mu_{p}}
$$

where it is understood that $p=0$ terms correspond to the $\operatorname{sum} \sum_{k} \hbar^{k} a_{k}$.

The product of elements in this algebra is given by the Weyl product rule

$$
\begin{aligned}
a \circ b & =\left.\exp \left(-\frac{\mathrm{i} \hbar}{2} \omega^{i j} \frac{\partial}{\partial y^{i}} \frac{\partial}{\partial z^{j}}\right) a(y, \hbar) b(z, \hbar)\right|_{z=y} \\
& =\sum_{k=0}^{\infty}\left(-\frac{\mathrm{i} \hbar}{2}\right)^{k} \frac{1}{k !} \omega^{i_{1} j_{1}} \ldots \omega^{i_{k} j_{k}} \frac{\partial^{k} a}{\partial y^{i_{1}} \ldots \partial y^{i_{k}}} \frac{\partial^{k} b}{\partial z^{j_{1}} \ldots \partial z^{j_{k}}},
\end{aligned}
$$

which is associative and independent of the particular choice of basis on the tangent space $T_{x}(M)$. The algebras $W_{x}$ constitute the fibers of the Weyl bundle $W$ of formal Weyl algebras, which is defined through the union of all fibers

$$
W=\bigcup_{x \in M} W_{x}
$$

Differential $q$-forms on $M$ with values in $W$ are sections of the bundle $W \otimes \Omega^{q}$ defined by it formal series

$$
a(x, y, \hbar)=\sum_{k, p \geq 0} \hbar^{k} a_{k, i_{1} \ldots 1_{p}, j_{1} \ldots j_{q}}(x) y^{i_{1}} \ldots y^{i_{p}} d x^{j_{1}} \wedge \ldots \wedge d x^{j_{q}}
$$

where the coefficients $a_{k, i_{1} \ldots 1_{p}, j_{1} \ldots j_{q}}(x)$ are covariant tensors fields symmetric in $i_{1} \ldots i_{p}$ and antisymmetric in $j_{1} \ldots j_{q}$ depending on a point in $M$. They constitute an algebra $C^{\infty}\left(W \otimes \Omega^{*}\right)$ under the usual Weyl product and the exterior product of forms, with unit identically equal to 1 . The total $\operatorname{degree} \operatorname{deg} a=2 k+p$ corresponding to the variables $\hbar$ 
and $y^{i}$ establishes a filtration within this algebra $W \otimes \Omega^{*} \supset W_{1} \otimes \Omega^{*} \supset W_{2} \otimes \Omega^{*} \supset \cdots$. For $q=0$ we have sections simply as formal series

$$
a(x, y, \hbar)=\sum_{k,|\alpha| \geq 0} \hbar^{k} a_{k, \alpha}(x) y^{\alpha},
$$

with $a_{k, \alpha}(x)$ covariant symmetric tensor fields on $M$. The bracket of two forms $a \in W \otimes \Omega^{q_{1}}$ and $b \in W \otimes \Omega^{q_{2}}$ takes the form

$$
[a, b]=a \circ b-(-1)^{q_{1} q_{2}} b \circ a .
$$

A form $a$ will be called central if for any $b \in C^{\infty}\left(W \otimes \Omega^{*}\right)$ the commutator (2.7) vanishes. The center of $C^{\infty}\left(W \otimes \Omega^{*}\right)$ consists exclusively of forms not containing $y$, which suggests a projection into the center by setting $y$ to zero. For $a \in C^{\infty}(W)$ we will denote such projection by

$$
\sigma(a)=a(x, 0, \hbar) .
$$

It is customary to define operators that rise and lower the rank of differential forms: $\delta$ : $C^{\infty}\left(W_{p} \otimes \Omega^{q}\right) \rightarrow\left(W_{p-1} \otimes \Omega^{q+1}\right)$ and $\delta^{*}: C^{\infty}\left(W_{p} \otimes \Omega^{q}\right) \rightarrow\left(W_{p+1} \otimes \Omega^{q-1}\right)$, and respectively lower and rise the degree of each term in the formal series. These fulfil the following properties

- $\delta^{2}=\left(\delta^{*}\right)^{2}=0$,

- $\delta(a \circ b)=\delta(a) \circ b+(-1)^{q} a \circ \delta(b)$, for $a \in \Omega^{q}$

- $a=\delta \delta^{-1} a+\delta^{-1} \delta a+a_{00}$,

where $a_{p q}$ denotes the homogeneous part of Weyl degree $p$ and cohomological degree $q$ of $a \in W \otimes \Omega^{q}$. Note that the last relation is similar to the Hodge-Rahm decomposition, yet $\delta$ is a pure algebraic operator on $M$ as we can see from its realization in local coordinates

$$
\delta a=d x^{k} \wedge \frac{\partial}{\partial y^{k}} a=-\frac{\mathrm{i}}{\hbar}\left[\omega_{i j} y^{i} d x^{j}, a\right] .
$$

To define a connection on the Weyl bundle one recalls there exists a symplectic connection on any symplectic manifold that is torsion free and preserves the symplectic structure

$$
\partial_{k} \omega_{i j}-\omega_{n l} \Gamma_{m k}^{n}-\omega_{k n} \Gamma_{m l}^{n}=0 .
$$

Using Darboux coordinates one can check that the connection symbols $\Gamma_{i j k}$ are completely symmetric $\Gamma_{[i j k]}=0$. The symplectic connection can be used to define a connection on the bundle $W \otimes \Omega^{*}$ by its action on elements of $C^{\infty}\left(W \otimes \Omega^{*}\right)$

$$
\nabla a=d x^{i} \wedge \nabla_{i} a
$$

in Darboux coordinates it reduces to

$$
\nabla a=d a+\frac{\mathrm{i}}{\hbar}[\Theta, a]
$$

where the connection symbols are $\Theta=\frac{1}{2} \Theta_{i j k} y^{i} y^{j} d x^{k}$. The properties of the symplectic Weyl bundle connection are 
- $\nabla(a \circ b)=(\nabla a) \circ b+(-1)^{q} a \circ(\nabla b)$, for $a \in \Omega^{q}$,

- for any $\phi \in \Omega^{q}, \nabla(\phi \wedge a)=d \phi \wedge a+(-1)^{q} \phi \wedge \nabla a$.

More general covariant derivatives $\mathcal{D}$ on the bundle may be considered by adding a one form $\gamma$ globally defined on $M$ with values in $W$,

$$
\mathcal{D} a=\nabla a+\frac{\mathrm{i}}{\hbar}[\gamma, a] .
$$

With this at hand the Weyl curvature may be defined as

$$
\Upsilon=R+\nabla \gamma+\frac{\mathrm{i}}{2 \hbar}[\gamma, \gamma]
$$

where $R$ is the curvature associated to the connection $\nabla$. The Weyl curvature satisfies the Bianchi identity

$$
\mathcal{D} \Upsilon=\nabla \Upsilon+\frac{\mathrm{i}}{\hbar}[\gamma, \Upsilon]=0 .
$$

Furthermore, for any section $a \in C^{\infty}\left(W \otimes \Omega^{*}\right)$

$$
\mathcal{D}^{2} a=\frac{\mathrm{i}}{\hbar}[\Upsilon, a] .
$$

In general, transitions on the bundle $T(M)$ will induce transitions on $W \otimes \Omega^{*}$. The infinitesimal gauge transformations on elements of $C^{\infty}\left(W \otimes \Omega^{*}\right)$ are expressed as automorphisms given by

$$
a \rightarrow a+[a, \lambda]
$$

with infinitesimal $\lambda \in C^{\infty}\left(W \otimes \Omega^{*}\right)$. The corresponding gauge transformations for the connections $\mathcal{D}$ are

$$
\mathcal{D} \rightarrow \mathcal{D}+\mathcal{D} \lambda
$$

consequently,

$$
\mathcal{D} a \rightarrow \mathcal{D} a+[\mathcal{D} a, \lambda]
$$

There exists a relation between the center and an Abelian subalgebra of the Weyl bundle that maps the Weyl product to the Moyal product through the projection (2.8). To establish such relation one first defines Abelian connections $\mathcal{D}_{A}$ as connections whose Weyl curvature is a central form in $C^{\infty}\left(W \otimes \Omega^{*}\right)$, i.e.,

$$
\left[\Omega_{A}, a\right]=0 \quad \forall a \in C^{\infty}\left(W \otimes \Omega^{*}\right) .
$$

An example of such connections may be expressed as

$$
\mathcal{D}_{A} a=\nabla a+\frac{\mathrm{i}}{\hbar}\left[\omega_{i j} y^{i} d x^{j}+r, a\right]
$$

with $\operatorname{deg} r \geqslant 3$. The Abelian subalgebra is the set $W_{A}=\left\{a \in W / \mathcal{D}_{A} a=0\right\}$ associated to $\mathcal{D}_{A}$. There is a one-to-one correspondence between the projections defined above (2.8) and 
elements of $W_{A}$. It is clear that for every $a \in W_{A}$ there is a projection projection to the center

$$
\sigma(a)=a_{00} .
$$

Now, given $a_{00}$ there is a unique element $a \in W_{A}$ with such projection. By means of this construction, from the Weyl product of two elements $a, b$ of $W_{A}$ we obtain a globally defined star product on $M$ which coincides with the Moyal product when $\omega_{i j}$ is constant and the symplectic connection is zero

$$
\sigma(a \circ b)=a_{00} \star b_{00} .
$$

\section{Weyl superalgebra bundles}

The main purpose of this work is the generalization of the Weyl bundle construction to superspace in order to study deformations of superfield algebras, which can then be used to extend supersymmetric field theories expressed in the superfield formalism. Following closely the known construction of Weyl bundle, we will need a symplectic structure $\omega$ defined on a supermanifold $\mathcal{M}$. In the following section we give the first steps toward such extension of deformation quantization, by defining this concepts and introducing a new Weyl product of superfields.

\subsection{Symplectic supermanifolds}

The structure underlying the concept of supermanifold ${ }^{1}$ is that of $\mathbb{R}^{p \mid q}$ space, i.e. the topological space $\mathbb{R}^{p}$ endowed with a sheaf $C^{\infty}\left[\theta^{1}, \theta^{2}, \ldots, \theta^{q}\right]$ of super $\mathbb{R}$-algebras freely generated over $C^{\infty}\left(\mathbb{R}^{p}\right)$ by the Graßmann $\left(\mathbb{Z}_{2}\right.$-graded) algebra basis $\left\{\theta^{1}, \theta^{2}, \ldots, \theta^{q}\right\}$. We will represent the set of superspace coordinates by

$$
z^{A}=\left(x^{m}, \theta^{\hat{\alpha}}\right), \quad m=1, \ldots p, \quad \hat{\alpha}=1, \ldots, q,
$$

where capital indices consist of the total set of superspace indices, which comprise lowercase $m, n, \ldots$ for standard spacetime directions, and $\hat{\alpha}, \hat{\beta}, \ldots$ for doted and undoted Graßmannodd indices. The anticommuting (odd) variables $\theta^{\hat{\alpha}}$ obey

$$
\left\{\theta^{\hat{\alpha}}, \theta^{\hat{\beta}}\right\}=0, \quad \hat{\alpha}, \hat{\beta}=1, \ldots, q .
$$

Like usual manifolds, that are locally isomorphic to $\mathbb{R}^{p}$, a supermanifold $\mathcal{M}$ of dimension $p \mid q$ consists of a topological space $M$ together with a sheaf of $\mathbb{R}$-algebras called the structure sheaf $\mathcal{O}_{\mathcal{M}}$, such that $\mathcal{M}$ is locally isomorphic to $\mathbb{R}^{p \mid q}$.

The differential geometry involving supermanifolds is quite similar to the classical one, having similar definitions and properties for super vector bundles, connections and actions of super Lie groups. The most significant difference arises from the existence of differential forms with arbitrary high degree, which hinders a straightforward generalization of integration. Nevertheless, after introducing the Berezinian, integration over supermanifolds can be easily defined.

\footnotetext{
${ }^{1}$ For an extensive review on the subject of supermanifolds from a mathematical point of view see 22 . The generalization of supermanifolds aimed at more physically inspired definitions can be found in 23, 24]
} 
A super vector bundle of rank $p \mid q$ over $\mathcal{M}$ is a sheaf of locally free $\mathcal{O}_{\mathcal{M}}$-supermodules $\mathcal{V}$ of dimension $p \mid q$. It can be also defined as a fibre bundle over $\mathcal{M}$ with typical fiber $\mathbb{R}^{p \mid q}$, having as structure group the super general linear group $G L(p \mid q)$. The first example of super vector bundle is the tangent bundle of a supermanifold, which can be constructed from the derivations of the superalgebra $\mathcal{O}_{\mathcal{M}}$ satisfying

$$
D(f g)=(D f) g+(-1)^{p(D) p(f)} f(D g), \quad \forall f, g \in \mathcal{O}_{\mathcal{M}} .
$$

where $p$ defines the $\mathbb{Z}_{2}$-grading of objects ( 0 for even, 1 for odd). The $\mathcal{O}$-supermodule of derivations of $\mathcal{O}_{\mathcal{M}}$ is a vector bundle of dimension $p \mid q$ called the tangent bundle $T(\mathcal{M})$, whose local basis consist of even $\partial / \partial x^{m}$ and odd $\partial / \partial \theta^{\hat{\alpha}}$ derivations. Sections of the tangent bundle are called vector fields and are locally written as

$$
Y=Y^{A} e_{A}=y^{m} \frac{\partial}{\partial x^{m}}+\eta^{\hat{\alpha}} \frac{\partial}{\partial \theta^{\hat{\alpha}}} .
$$

The set of vector fields $\operatorname{Vect}(\mathcal{M})$ is a super Lie algebra.

The cotangent bundle $\Omega_{\mathcal{M}}^{1}$ of $\mathcal{M}$ is the dual of $T(\mathcal{M})$, whose basis we denote by $d z^{A}=\left(d x^{m}, d \theta^{\hat{a}}\right)$. One can interpret the duality pairing as an inner product $\langle\cdot, \cdot\rangle: T(\mathcal{M}) \otimes$ $\Omega_{\mathcal{M}}^{1} \longrightarrow \mathcal{O}_{\mathcal{M}}$, satisfying

$$
\langle f Y, g \omega\rangle=(-1)^{p(Y) p(g)} f g\langle Y, \omega\rangle, \quad \forall f, g \in \mathcal{O}_{\mathcal{M}} .
$$

In the local basis, such product can be obtained by linearity from

$$
\left\langle\frac{\partial}{\partial z^{A}}, d z^{B}\right\rangle=\delta_{A}^{B}
$$

The wedge product of 1 -forms is defined through

$$
d z^{A} \wedge d z^{B}=-(-1)^{p(A) p(B)} d z^{B} \wedge d z^{A}
$$

where $p(A)$ indicates the parity of an object with index $A$. One can linearly extend this definition to the exterior product $\wedge: \Omega_{\mathcal{M}}^{k} \times \Omega_{\mathcal{M}}^{l} \longrightarrow \Omega_{\mathcal{M}}^{k+l}$ of $k$-forms with $l$-forms, providing the set of all forms with the structure of a superalgebra which is called the exterior superalgebra $\Omega_{\mathcal{M}}^{*} \equiv \wedge^{*} \Omega_{\mathcal{M}}^{1}$. Note that in contrast with the wedge product of forms, supercoordinates and superfield components conform supercommutative superalgebras like

$$
z^{A} z^{B}=(-1)^{p(A) p(B)} z^{B} z^{A}
$$

as the concepts of cohomological degree of a form, and that of Graßmann parity are independent.

The derivative $d: \mathcal{O}_{\mathcal{M}} \longrightarrow \Omega_{\mathcal{M}}^{1}$ defined by

$$
\langle Y, d f\rangle=Y(f)
$$

extends uniquely to the exterior derivative $d=d z^{A} \partial / \partial z^{A}$ on the exterior superalgebra and satisfies

$$
d^{2}=0, \quad d(\omega \chi)=d \omega \wedge \chi+(-1)^{p} \omega \wedge d \chi, \quad \omega \in \Omega_{\mathcal{M}}^{p}
$$


From here onwards we will take $p=2 n$ even and $q=4 N$ representing the physically relevant set of odd variables, thus studying only the supermanifold $\mathbb{R}^{2 n \mid 4 N}$. In $4 \mid 4$ dimensions, for example we have $A=(m, \alpha, \dot{\alpha})$. i.e. $z^{A}=\left(x^{m}, \theta^{\alpha}, \bar{\theta}_{\dot{\alpha}}\right)$, with $\alpha=1,2, \dot{\alpha}=\dot{1}, \dot{2}$. The notation we use is consistent with that of Wess and Bagger 25] for differential forms in superspace.

A symplectic supermanifold is a $2 n \mid 4 N$ dimensional supermanifold $\mathcal{M}$ endowed with a closed, non-degenerated two-form $\omega$ called the symplectic superform. In coordinates,

$$
\omega=\omega_{A B} d z^{A} \wedge d z^{B} \text {. }
$$

The Darboux theorem [27, 29, 28] states that there exist local coordinates on the supermanifold where the symplectic superform $\omega$ has the following block shape

$$
\omega_{A B}=\left(\begin{array}{cc}
\omega_{m n} & 0 \\
0 & \omega_{\hat{\alpha} \hat{\beta}}
\end{array}\right),
$$

with Graßmann-even components having definite symmetry under index exchange $\omega_{m n}=$ $-\omega_{n m}$ and $\omega_{\hat{\alpha} \hat{\beta}}=\omega_{\hat{\beta} \hat{\alpha}}$. The symplectic superform can be used to rise and lower indices. Symplectic supermanifolds always admit a torsionless affine connection preserving the symplectic superform, called (super)symplectic connection. We will denote the connection by $\nabla$, and define its coefficients $\Gamma_{B C}^{A}$ by its action on the tangent base

$$
\nabla_{e_{A}} e_{B}=\nabla_{A} e_{B}=\Gamma_{A B}^{C} e_{C}
$$

Such coefficients can be found from the Leibnitz property of the covariant derivative

$$
\nabla_{Y_{1}}\left(\omega\left(Y_{2}, Y_{3}\right)\right)=\left(\nabla_{Y_{1}} \omega\right)\left(Y_{2}, Y_{3}\right)+\omega\left(\nabla_{Y_{1}} Y_{2}, Y_{3}\right)+\omega\left(Y_{2}, \nabla_{Y_{1}} Y_{3}\right)
$$

The conditions for the vanishing of the torsion in superspace can be derived from its very definition in terms of vector fields $Y_{1}$ and $Y_{2}$

$$
T=\nabla_{Y_{1}} Y_{2}-\nabla_{Y_{2}} Y_{1}-\left[Y_{1}, Y_{2}\right],
$$

resulting in the following components

$$
\begin{aligned}
T_{m n}^{A} & =\Gamma_{m n}^{A}-\Gamma_{n m}^{A} \\
T_{m \hat{\alpha}}^{A} & =\Gamma_{m \hat{\alpha}}^{A}-\Gamma_{\hat{\alpha} m}^{A} \\
T_{\hat{\alpha} \hat{\beta}}^{A} & =\Gamma_{\hat{\alpha} \hat{\beta}}^{A}+\Gamma_{\hat{\beta} \hat{\alpha}}^{A}
\end{aligned}
$$

A torsion free affine connection associated to our symplectic superform is defined by the equation

$$
\nabla_{X} \omega\left(Y_{1}, Y_{2}\right)=\nabla_{X}\left[\omega\left(Y_{1}, Y_{2}\right)\right]-\omega\left(\nabla_{X} Y_{1}, Y_{2}\right)-\omega\left(Y_{1}, \nabla_{X} Y_{2}\right)=0
$$

In Darboux coordinates, the resulting conditions on the connection coefficients (3.13) are

$$
\begin{array}{r}
\omega_{k l} \Gamma_{m n}^{k}+\omega_{n k} \Gamma_{m l}^{k}=0 \\
\omega_{\hat{\alpha} \hat{\beta}} \Gamma_{m n}^{\hat{\beta}}-\omega_{n k} \Gamma_{m \hat{\alpha}}^{k}=0 \\
\omega_{\hat{\alpha} \hat{\beta}} \Gamma_{\hat{\delta} \hat{\gamma}}^{\hat{\beta}}+\omega_{\hat{\gamma} \hat{\beta}} \Gamma_{\hat{\delta} \hat{\alpha}}^{\hat{\beta}}=0 \\
\omega_{m n} \Gamma_{\hat{\alpha} \hat{\beta}}^{n}-\omega_{\hat{\beta} \hat{\delta}} \Gamma_{\hat{\alpha} m}^{\hat{\delta}}=0
\end{array}
$$


Following (3.16), the torsionless components of the symplectic connection are

$$
\begin{aligned}
\Gamma_{l n m} & =\Gamma_{n l m} \\
\Gamma_{l \hat{\alpha} n} & =\Gamma_{n \hat{\alpha} l} \\
\Gamma_{l \hat{\alpha} \hat{\beta}} & =\Gamma_{\hat{\beta} \hat{\alpha} l} \\
\Gamma_{\hat{\gamma} \hat{\alpha} \hat{\beta}} & =-\Gamma_{\hat{\beta} \hat{\gamma} \hat{\alpha}}
\end{aligned}
$$

a completely symmetric object

a completely symmetric object

antisymmetric in 1,2 positions but symmetric in 2,3

a completely antisymmetric object

\subsection{Weyl superalgebra bundle through a new associative superproduct}

We will consider a symplectic supermanifold $(\mathcal{M}, \omega)$ locally isomorphic to $\mathbb{R}^{4 \mid 4}$ representing $N=1$ symplectic superspace, as most results are easily generalized to extended superspace. Local coordinates of $z \in \mathcal{M}$, and the components of a generic vector $Y \in T_{z}(\mathcal{M})$ in tangent space, are to be denoted $z^{A}=\left(x^{m}, \theta^{\alpha}, \bar{\theta}^{\dot{\alpha}},\right)$ and $Y^{A}=\left(y^{m}, \eta^{\alpha}, \bar{\eta}^{\dot{\alpha}}\right)$ respectively, where $\theta^{\hat{\alpha}}, \eta^{\hat{\alpha}}$ are Graßmann-odd. Without loss of generality we take the symplectic tensor defining the symplectic structure over fibers of $T(\mathcal{M})$ to have block diagonal components $\omega_{A B}$ as in (3.12).

A Weyl superalgebra $\mathcal{W}_{z}$ defined locally on the tangent superspace $T_{z}(\mathcal{M})$ is an associative superalgebra over $\mathbb{C}$, its elements being formal series on the parameter $\hbar$

$$
f(Y, \hbar)=\sum_{k, p} \hbar^{k} f_{k, A_{1}, \ldots, A_{p}} Y^{A_{1}} \cdots Y^{A_{p}}
$$

Taking advantage of the nilpotent nature of odd coordinates, we can collect the terms in the formal series into a superfield

$$
f(Y, \hbar)=\phi\left(y^{m}, \hbar\right)+\eta^{\alpha} \xi_{\alpha}\left(y^{m}, \hbar\right)+\cdots,
$$

whose components are elements of a standard Weyl algebra

$$
\phi\left(y^{m}, \hbar\right)=\sum_{k, p} \hbar^{k} \phi_{k, m_{1}, \ldots, m_{p}} y^{m_{1}} \cdots y^{m_{p}} .
$$

The component fields in (3.21) will have tensor or spinor indices, and Graßmann parity inherited from coefficients in the formal series $(3.20)$. An equivalent description is obtained by taking a formal series in even coordinates

$$
f(Y, \hbar)=\sum_{k, p} \hbar^{k} f_{k, m_{1}, \ldots, m_{p}} y^{m_{1}} \cdots y^{m_{p}},
$$

whose coefficients are superfields of tangent space odd coordinates.

$$
f_{k, m_{1}, \ldots, m_{p}}=\phi_{k, m_{1}, \ldots, m_{p}}+\eta^{\alpha} \xi_{\alpha, k, m_{1}, \ldots, m_{p}}+\cdots
$$

The vector bundle corresponding to the sheaf of Weyl superalgebras is the Weyl superalgebra bundle $\mathcal{W}$, whose sections are functions of the symplectic base space as in

$$
f(z, Y, \hbar)=\sum_{k, p} \hbar^{k} f_{k, A_{1}, \ldots, A_{p}}(z) Y^{A_{1}} \cdots Y^{A_{p}},
$$


or equivalently, superfields like (3.20) with components having non constant coefficients $f_{k, m_{1}, \ldots, m_{p}}(z)$.

A Weyl superalgebra can be then defined by means of the following Weyl product,

$$
f \odot g=\left.\exp \left(-\frac{\mathrm{i} \hbar}{2} \omega^{M N} \frac{\partial}{\partial Y^{M}} \frac{\partial}{\partial \tilde{Y}^{N}}\right) f(Y, \hbar) g(\tilde{Y}, \hbar)\right|_{\tilde{Y}=Y} .
$$

Since this product should preserve the degree, we propose $\operatorname{deg}(\eta)=1$. Similar structures that appeared in the context of anti-Poisson brackets on Graßmann algebras [26] were developed further into a Fedosov-type procedure on the dual Graßmann algebra bundle of a given vector bundle 19, 20]. Note that the product (3.25) differs from such antiMoyal products as it acts on the tangent superspace $T(\mathcal{M})$, thus protecting super Poincaré symmetry on $\mathcal{M}$ from breaking. In Darboux coordinates [27, 28] the Poisson structure $\omega^{M N}$ becomes block diagonal allowing a separation of the sum inside the exponential into a purely bosonic and a purely fermionic part. Each term in turn, as a bilinear operator, is even and commutes with the others. For instance

$$
\left[\omega^{m n} \frac{\partial}{\partial y^{m}} \frac{\partial}{\partial \tilde{y}^{n}}, \omega^{a b} \frac{\partial}{\partial \eta^{a}} \frac{\partial}{\partial \tilde{\eta}^{b}}\right]=0
$$

which is also true for any other combination of indices (note that a term with mixed indices $\omega^{m a}$ is absent). This allow us to split the exponential

$$
\exp \left(-\frac{\mathrm{i} \hbar}{2} \omega^{M N} \frac{\partial}{\partial Y^{M}} \frac{\partial}{\partial \tilde{Y}^{N}}\right)=\exp \left(-\frac{\mathrm{i} \hbar}{2} \omega^{m n} \frac{\partial}{\partial y^{m}} \frac{\partial}{\partial \tilde{y}^{n}}\right) \exp \left(-\frac{\mathrm{i} \hbar}{2} \omega^{a b} \frac{\partial}{\partial \eta^{a}} \frac{\partial}{\partial \tilde{\eta}^{b}}\right)
$$

and expand the Weyl superproduct as the nested application of two products: The usual Weyl rule for standard spacetime,

$$
f \circ g=\left.\exp \left(-\frac{\mathrm{i} \hbar}{2} \omega^{m n} \frac{\partial}{\partial y^{m}} \frac{\partial}{\partial \tilde{y}^{n}}\right) f(Y, \hbar) g(\tilde{Y}, \hbar)\right|_{\tilde{Y}=Y}
$$

and a new one for the fermionic coordinates

$$
f \bigcirc g=\left.\exp \left(-\frac{\mathrm{i} \hbar}{2} \omega^{a b} \frac{\partial}{\partial \eta^{a}} \frac{\partial}{\partial \tilde{\eta}^{b}}\right) f(Y, \hbar) g(\tilde{Y}, \hbar)\right|_{\tilde{Y}=Y} .
$$

Though both this products are formal series of the exponential

$$
f \circ g=\sum_{k=0}^{\infty}\left(-\frac{\mathrm{i} \hbar}{2}\right)^{k} \frac{1}{k !} \omega^{m_{1} n_{1}} \ldots \omega^{m_{k} n_{k}} \frac{\partial^{k} f}{\partial y^{m_{1}} \ldots \partial y^{m_{k}}} \frac{\partial^{k} g}{\partial \tilde{y}^{n_{1}} \ldots \partial \tilde{y}^{n_{k}}},
$$

in the fermionic case the series is truncated due to nilpotency of odd derivations. Nesting the products $\circ$ and $\bigcirc$ can be understood as the replacement of the standard product in the formal series of o with the product rule of $\bigcirc$, that is

$$
f \odot g=\sum_{k=0}^{\infty}\left(-\frac{\mathrm{i} \hbar}{2}\right)^{k} \frac{1}{k !} \omega^{m_{1} n_{1}} \ldots \omega^{m_{k} n_{k}} \frac{\partial^{k} f}{\partial y^{m_{1}} \ldots \partial y^{m_{k}}} \bigcirc \frac{\partial^{k} g}{\partial \tilde{y}^{n_{1}} \ldots \partial \tilde{y}^{n_{k}}} .
$$


Weyl products for the purely bosonic case (only $\omega^{m n} \neq 0$ ) are associative. Including the bosonic and the full fermionic blocks into the form $\omega^{M N}$ will lead to an associative product as a result of nesting the $\bigcirc$ products already known to be associative. As the defined product is covariant, a completely general proof of associativity can be worked out in Darboux coordinates. This can be verified directly by expanding the product $f \bigcirc(g \bigcirc h)$ for the fermionic part exclusively. One can see why this work by looking at the particular case with only two fermionic coordinates $Y^{A}=\left(y^{m}, \eta^{1}, \eta^{2}\right)$, which has a particular importance in the description of the Weyl bundle over chiral superspace having precisely such coordinates. The Weyl rule in this setup will looks like

$$
f \bigcirc g=f g+\left(-\frac{\mathrm{i} \hbar}{2}\right) \omega^{\alpha \beta}(-1)^{p(f)} \partial_{\alpha} f \partial_{\beta} g-\frac{1}{2}\left(-\frac{\mathrm{i} \hbar}{2}\right)^{2} \omega^{\alpha \beta} \omega^{\gamma \delta} \partial_{\alpha \gamma} f \partial_{\beta \delta} g,
$$

where we use the standard spinor index notation for the odd variables $\alpha=1,2$ and the usual shorthand $\partial_{\alpha}=\frac{\partial}{\partial \eta^{\alpha}}$ to denote derivation respect to $Y^{\alpha}=\eta^{\alpha}$ (with its straightforward extension $\partial_{\alpha \beta}=\partial_{\alpha} \partial_{\beta}$ ). Note that the series is truncated to second order, which facilitates direct verification of the associativity

$$
\begin{aligned}
(f \bigcirc g) \bigcirc h= & f g h \\
& +\left(-\frac{\mathrm{i} \hbar}{2}\right) \omega^{\alpha \beta}\left[(-1)^{p(f g)} \partial_{\alpha} f g \partial_{\beta} h+(-1)^{p(g)} f \partial_{\alpha} g \partial_{\beta} h+(-1)^{p(f)} \partial_{\alpha} f \partial_{\beta} g h\right] \\
& -\frac{1}{2}\left(-\frac{\mathrm{i} \hbar}{2}\right)^{2} \omega^{\alpha \beta} \omega^{\gamma \delta}\left[\partial_{\alpha \gamma} f g \partial_{\beta \delta} h+f \partial_{\alpha \gamma} g \partial_{\beta \delta} h+\partial_{\alpha \gamma} f \partial_{\beta \delta} g h\right. \\
& \left.-2(-1)^{p(f g)} \partial_{\alpha} f \partial_{\beta \gamma} g \partial_{\delta} h-2(-1)^{p(g)} \partial_{\gamma \alpha} f \partial_{\beta} g \partial_{\delta} h+(-1)^{p(f)} \partial_{\alpha} f \partial_{\gamma} g \partial_{\beta \delta} h\right] \\
= & f \bigcirc(g \bigcirc h) .
\end{aligned}
$$

A $\bigcirc$ product involving $4 N$ odd variables, as required by more general $N$-supersymmetric theories, is obtained by repeatedly nesting products as (3.32) above, resulting in an associative product with a truncated expansion of order $4 N$. If we include the standard o product in Darboux coordinates one also concludes that this product is associative in general, from the covariant character of $\odot$.

The Weyl superalgebra is easily extended to include $q$-superforms on $\mathcal{M}$, that is, sections on $\mathcal{W} \otimes \Omega^{*}$ expressed as

$$
f(z, Y, \hbar)=\sum_{k, p} \hbar^{k} f_{k, A_{1}, \ldots, A_{p}, B_{1} \ldots, B_{q}}(z) Y^{A_{1}} \cdots Y^{A_{p}} d z^{B_{1}} \wedge \ldots \wedge d z^{B_{q}} .
$$

These differential forms constitute an algebra with multiplication defined by the combined exterior superproduct of forms given in $\S 3.1$ together with the Weyl product acting on tangent superspace variables.

The commutator of 2 superforms $f \in \mathcal{W} \otimes \Omega^{q_{1}}$ and $g \in \mathcal{W} \otimes \Omega^{q_{2}}$ is

$$
[f, g]=f \odot g-(-1)^{q_{1} q_{2}}(-1)^{p(f) p(g)} g \odot f .
$$

A central superform $f$ is such that for any $g \in \mathcal{W} \otimes \Omega^{*},[f, g]=0$. In (3.35) the wedge exterior product $\wedge$ acting on forms is understood. 


\section{Superspace deformations}

\subsection{Abelian subalgebra and deformations}

In the purely bosonic case the Weyl product is linked to deformations on the base space through a correspondence between $C^{\infty}(M)$ and the Abelian subalgebra that, in Darboux coordinates, results in the well known Moyal product, as was proved in [15]. In this section we will show that this is also the case for the Weyl superproduct, which for the chiral case give us precisely the nonanticonmutative product extensively studied in this decade, see for example [3, 6, 7].

We start with the concept of Abelian connection. A connection $\mathcal{D}_{A}$ on the Bundle $\mathcal{W}$ is called Abelian if for any section $f \in \mathcal{W} \otimes \Omega^{*}$

$$
\mathcal{D}_{A}^{2} f=0
$$

Inspired by Fedosov construction, we will lift the supersymplectic connection defined in $\S 3.1$ to the Weyl superalgebra bundle

$$
\mathcal{D}=d z^{A} \wedge \nabla_{A} f
$$

$\mathcal{D}$ is a covariant derivative acting on $C^{\infty}\left(\mathcal{W} \otimes \Omega^{*}\right)$ and obeying the following properties

$$
\begin{aligned}
\mathcal{D}(f \odot g) & =\mathcal{D} f \odot g+(-1)^{q_{1}+p(f)} f \odot \mathcal{D} g \\
\mathcal{D}[f, g] & =[\mathcal{D} f, g]+(-1)^{q_{1}+p(f)}[f, \mathcal{D} g]
\end{aligned}
$$

Another important operators involved in the construction of the Abelian connection are $\delta: \mathcal{W}_{p} \otimes \Omega^{q} \rightarrow \mathcal{W}_{p-1} \otimes \Omega^{q+1}$ that raises the rank of forms and acts as a sort of exterior superderivative, and $\delta^{*}: \mathcal{W}_{p} \otimes \Omega^{q} \rightarrow \mathcal{W}_{p+1} \otimes \Omega^{q-1}$, that lowers the rank of forms and acts as a contraction operator. In coordinate basis they are realized as

$$
\delta f=d z^{A} \wedge \frac{\partial}{\partial Y^{A}} f \quad \quad \delta^{*} f=\mathrm{i} Y^{A} \wedge \frac{\partial}{\partial z^{A}} f,
$$

Using (3.8), (3.7) and

$$
d z^{A} \wedge Y^{B}=(-1)^{p(Y) p(d z)},
$$

and observing their action on each term in the formal series

$$
f_{k, A_{1}, \ldots, A_{p}, B_{1}, \ldots, B_{q}} Y^{A_{1}} \cdots Y^{A_{p}} d z^{B_{1}} \wedge \cdots \wedge d z^{B_{q}},
$$

one can check their properties

$$
\begin{aligned}
\delta^{2} & =0, \\
\left(\delta^{*}\right)^{2} & =0, \\
\delta(f \odot g) & =\delta f \odot g+(-1)^{p(f)+q} f \odot \delta g, \\
f & =\delta \delta^{-1} f+\delta^{-1} \delta f+f_{00} .
\end{aligned}
$$


Here $f_{p q}$ is the coefficient with $p$ times $Y$ and $q$ times $d z$, so that $(p+q) \delta^{-1} f_{p q}=\delta^{*} f_{p q}$.

Given the supersymplectic connection $\mathcal{D}$, there always exists an Abelian connection

$$
\mathcal{D}_{A} \cdot=\mathcal{D} \cdot+\frac{\mathrm{i}}{\hbar}\left[\left(\omega_{A B} Y^{A} Y^{B}+r\right), \cdot\right]
$$

where $r \in C^{\infty}\left(\mathcal{W}_{3} \otimes \Omega^{1}\right)$ satisfies $\delta^{-1} r=0$.

The proof follows the corresponding one in Fedosov [15, §5.2], where mainly the properties of $\delta, \delta^{-1}$ and degree counting are used. The arguments are exactly the same. The curvature of the Abelian connection is

$$
F=-\frac{1}{2} \omega_{A B} d z^{A} \wedge d z^{B}+R-\delta r+\mathcal{D} r+\frac{\mathrm{i}}{\hbar} r^{2}
$$

We consider then $r$ satisfying

$$
\delta r=R+\mathcal{D} r+\frac{\mathrm{i}}{\hbar} r^{2},
$$

which ensures that $\mathcal{D}_{A}$ is Abelian. Eq (4.12) has a unique solution belonging to $C^{\infty}\left(\mathcal{W}_{3} \otimes\right.$ $\Omega^{1}$ ) and satisfying $\delta^{-1} r=0$. The main point in Fedosov's proof, which can be extended to the supersymplectic case, is to show that

$$
r=\delta^{-1}\left(R+\mathcal{D} r+\frac{\mathrm{i}}{\hbar} r^{2}\right)
$$

has a unique solution belonging to $C^{\infty}\left(\mathcal{W}_{3} \otimes \Omega^{1}\right)$. This follows from the iteration procedure defined by

$$
r_{n+1}=\delta^{-1} R+\delta^{-1}\left(\mathcal{D} r_{n}+\frac{\mathrm{i}}{\hbar} r_{n}^{2}\right), \quad n=0,1,2, \ldots
$$

From degree counting one obtains $\operatorname{deg}\left(r_{n}-r_{n+1}\right) \geq n+3$ implying that the terms for any fixed degree of the sequence are the same for large enough $n$. There exists then a unique solution in $C^{\infty}\left(\mathcal{W}_{3} \otimes \Omega^{1}\right)$. It is then straightforward to show that this solution satisfies (4.12) as $\delta^{-1} r=0$ holds by construction. Uniqueness arises from acting on (4.12) with $\delta^{-1}$ and using the above property of $r$.

With this at hand we propose an Abelian connection of the simplest form

$$
\mathcal{D}_{A} f=\mathcal{D} f-\delta f
$$

Once we have the Abelian connection, we use it to find the elements of the Abelian $\operatorname{sub}$ (super)algebra $\mathcal{W}_{A}=\left\{f \in \mathcal{W}: \mathcal{D}_{A} f=0\right\}$. There is a one-to-one correspondence between the set of solutions to the equation $\mathcal{D}_{A} f=0$ and functions on the base space $\mathcal{M}$, that relates the Weyl product to a deformation on the base space. A proof of this correspondence is a straightforward generalization of the original theorem by Fedosov (5.2.4 in 15).

Let us consider a section $f \in C^{\infty}(\mathcal{W}), f=f(x, \theta, y, \eta)$, and focus in the Abelian subalgebra $\mathcal{D}_{A} f=0$. As in Fedosov [15] we take

$$
\delta f=\left(\mathcal{D}_{A}+\delta\right) f
$$


being $f$ a 0 -form for which $\delta^{-1} f=0$ holds, the Hodge-De Rham decomposition results in

$$
f=\left.f\right|_{y=\eta=0}+\delta^{-1}\left(\mathcal{D}_{A}+\delta\right) f .
$$

Given $\left.f\right|_{y=\eta=0}=f(x, \theta, 0,0)$, as $\delta^{-1}$ raises degree, an iteration procedure yields existence and uniqueness of the solution. The bijection then follows from (4.17).

Considering bosonic coordinates only, it is known that the deformation induced in base space by a Weyl product in Darboux coordinates is precisely the Moyal product [15, 9]. More succinctly, let $\sigma: W_{A} \longrightarrow C^{\infty}(M)[[\hbar]]$ be the projection to the center that maps $\sigma: a \mapsto a_{00}$ and $\sigma: b \mapsto b_{00}$, then

$$
\sigma(a \circ b)=a_{00} \star b_{00},
$$

where $\star$ stands for the Moyal product.

When general sections in the Abelian subalgebra defined by an arbitrary Abelian connection are considered, the projection $\varsigma: \mathcal{W}_{A} \longrightarrow C^{\infty}(\mathcal{M})[[\hbar]]$ to the center, will take the $\odot$ superproduct to a nonanticommutative $\star$ product

$$
\varsigma(f \circ g)=f_{00} \star g_{00},
$$

where $f, g, f_{00}$ and $g_{00}$ are now superfields. The structure of such non(anti)commutative * product generalizes the usual treatment given in the literature [21], as the associated non(anti)commuting parameter is actually a function of the base supermanifold variables reflecting the curvature of the Abelian connection.

In the next section we will obtain the nonanticommutative $\star$ product for the flat case, proposed in [4, 6, 7] as a projection of the $\odot$ superproduct to the base space using Darboux chiral coordinates in superspace.

\section{Nonanticommutative $Q$-deformations from Weyl superproduct}

In this section we will restrict ourselves to functions $f \in C^{\infty}\left(\mathcal{W} \otimes \Omega^{0}\right)$ whose coefficients are chiral superfields, i.e. $\bar{D}^{\dot{\alpha}} f=0$, where $\bar{D}^{\dot{\alpha}}$ stands for the supersymmetric derivative in the base space. In order to obtain nonanticommutativity in the base space, we will drop $\omega^{m n}$ and $\omega^{\dot{\alpha} \dot{\beta}}$ terms. The Weyl rule is directly generalized to this setup in Darboux coordinates

$$
f \odot g=f \bigcirc g=f g+\left(-\frac{\mathrm{i} \hbar}{2}\right) \omega^{\alpha \beta}(-1)^{p(f)} \partial_{\alpha} f \partial_{\beta} g-\frac{1}{2}\left(-\frac{\mathrm{i} \hbar}{2}\right)^{2} \omega^{\alpha \beta} \omega^{\gamma \delta} \partial_{\alpha \gamma} f \partial_{\beta \delta} g,
$$

Now we consider the following Abelian connection

$$
\mathcal{D}_{A} f=\mathcal{D} f-\delta f=\mathcal{D} f+\frac{\mathrm{i}}{\hbar}\left[\omega_{\alpha \beta} \eta^{\alpha} d \theta^{\beta}, f\right]
$$

and build the Abelian sub algebra of Graßmann-even superfields from the condition $\mathcal{D}_{A} f=$ 0. The symplectic connection can be build using the super Poincaré generator

$$
\mathcal{D}=d \theta^{\alpha} \wedge Q_{\alpha}
$$


in chiral coordinates, we have

$$
\mathcal{D}_{A} f=d \theta^{\alpha} \wedge \frac{\partial}{\partial \theta^{\alpha}} f+\omega^{\alpha \gamma} \omega_{\alpha \beta} d \theta^{\beta} \frac{\partial}{\partial \eta^{\gamma}} f
$$

Focusing on Graßmann-even superfields on the generalization of the Weyl bundle

$$
f=f_{0}+\hbar f_{1 \alpha} \eta^{\alpha}+\hbar^{2} f_{2 \alpha \beta} \eta^{\alpha} \eta^{\beta}
$$

the Abelian condition reduces to the following set of equations

$$
\begin{aligned}
\frac{\partial f_{0}}{\partial \theta^{\gamma}}+h \omega^{\alpha \beta} \omega_{\alpha \gamma} f_{1 \beta} & =0 \\
\frac{\partial f_{1 \alpha}}{\partial \theta^{\gamma}}+\hbar \omega^{\delta \beta} \omega_{\delta \gamma} 2 f_{2} \varepsilon_{\beta \alpha} & =0 \\
\frac{\partial f_{2}}{\partial \theta^{\gamma}} & =0 .
\end{aligned}
$$

As the symplectic form is everywhere not degenerate, $\omega^{\alpha \beta} \omega_{\alpha \gamma}=\delta_{\gamma}^{\beta}$, we easily find the general form of an element of the Abelian subalgebra to be

$$
f=f_{0}-Q_{\alpha} f_{0} \eta^{\alpha}+\frac{1}{4} Q^{\alpha} Q_{\alpha} f_{0}\left(\eta^{\beta} \eta_{\beta}\right)
$$

The product of two elements of this subalgebra is projected back to the nonanticommutative Moyal product [四, 6, 可]

$$
\begin{aligned}
f \bigcirc g & =f_{0} g_{0}-\frac{\mathrm{i} \hbar}{2} \omega^{\alpha \beta} Q_{\alpha} f_{0} Q_{\beta} g_{0}-\frac{1}{2}\left(-\frac{\mathrm{i} \hbar}{2}\right)^{2} \omega^{2} Q^{\alpha} Q_{\alpha} f_{0} Q^{\beta} Q_{\beta} g_{0}+\mathrm{O}(\eta), \\
\sigma(f \bigcirc g) & =f_{0} \star g_{0} .
\end{aligned}
$$

A first attempt to introduce curvature in the previous setup is to include nonzero connection coefficients $\Gamma_{\alpha \beta}^{\gamma}$

$$
\left(\nabla_{\eta} \omega\right)(\chi, \psi)=\eta^{\alpha} \chi^{\beta} \psi^{\gamma}\left(\partial_{\alpha} \omega_{\beta \gamma}+\Gamma_{\gamma \alpha \beta}+\Gamma_{\beta \alpha \gamma}\right)
$$

for Graßmann vector fields $\eta, \chi$ and $\psi$. i.e.

$$
\eta=\eta^{\alpha} \frac{\partial}{\partial \theta^{\alpha}}
$$

The antisymmetric connection coefficients are defined up to an arbitrary completely antisymmetric tensor $\Theta_{\alpha \beta \gamma}$.

On sections $a$ of the superbundle, the connection is lifted to

$$
\mathcal{D} a=d \theta^{\alpha} \wedge \nabla_{\alpha} a=d a+\frac{\mathrm{i}}{h}[\Theta, a],
$$

with

$$
\Theta=\frac{1}{2} \Theta_{\alpha \beta \gamma} \eta^{\alpha} \eta^{\beta} d \theta^{\gamma}
$$


An Abelian connection can then be constructed via

$$
\mathcal{D}_{A} a=d a+\frac{\mathrm{i}}{h}\left[\omega_{\alpha \beta} \eta^{\alpha} d \theta^{\beta}+\frac{1}{2} \Theta_{\alpha \beta \gamma} \eta^{\alpha} \eta^{\beta} d \theta^{\gamma}, a\right]_{\circ} .
$$

The associated Weyl subalgebra is obtained following the same procedure as for (5.6), leading to an analogous correspondence between undeformed sections and Abelian ones, now including a more general covariant derivative. The projection of the Weyl product results in an expression which is formally the same as before (5.8) due to nilpotency of the Grassmann basis, which truncates the series to second order in the deformation, preventing the appearance of terms involving the curvature. This product does not correspond to the standard Moyal product, as it includes connection coefficients. Explicit expressions for more general non(anti)commutative products following the approach introduced in this work will be presented in a forthcoming paper, this includes the description of $D$-deformations.

\section{Acknowledgments}

This work was partially supported by the USB-DID S1 grants (A. De Castro and L. Quevedo). The work of A. Restuccia is supported by a grant of the Albert Einstein Institute, Max Planck Institute.

\section{A. Conventions}

Spinor algebra follows the convention in Wess-Bagger

$$
\begin{array}{rlrl}
\eta^{\alpha} \eta^{\beta} & =-\frac{1}{2} \varepsilon^{\alpha \beta}(\eta \eta), & \eta_{\alpha} \eta_{\beta} & =+\frac{1}{2} \varepsilon_{\alpha \beta}(\eta \eta), \\
\bar{\eta}_{\dot{\alpha}} \bar{\eta}_{\dot{\beta}}=-\frac{1}{2} \varepsilon_{\dot{\alpha} \dot{\beta}}(\bar{\eta} \bar{\eta}), & \bar{\eta}^{\dot{\alpha}} \bar{\eta}^{\dot{\beta}}=+\frac{1}{2} \varepsilon^{\dot{\alpha} \dot{\beta}}(\bar{\eta} \bar{\eta}) .
\end{array}
$$

But with a more comfortable definition for the derivative

$$
\begin{aligned}
\partial_{\alpha} & =\frac{\partial}{\partial \eta^{\alpha}}, & \bar{\partial}^{\dot{\alpha}} & =\frac{\partial}{\partial \bar{\eta}_{\dot{\alpha}}} \\
\partial^{\alpha} & =-\frac{\partial}{\partial \eta_{\alpha}}=\varepsilon^{\alpha \beta} \partial_{\beta}, & \bar{\partial}_{\dot{\alpha}} & =-\frac{\partial}{\partial \bar{\eta}^{\dot{\alpha}}}=\varepsilon_{\dot{\alpha} \dot{\beta}} \bar{\partial}^{\dot{\beta}} .
\end{aligned}
$$

\section{References}

[1] V. Schomerus, D-branes and deformation quantization, JHEP 06 (1999) 030 hep-th/9903205.

[2] M.R. Douglas and C.M. Hull, D-branes and the noncommutative torus, JHEP 02 (1998) 008 hep-th/9711165.

[3] N. Seiberg, Noncommutative superspace, $N=1 / 2$ supersymmetry, field theory and string theory, JHEP 06 (2003) 010 hep-th/0305248.

[4] N. Seiberg and E. Witten, String theory and noncommutative geometry, JHEP 09 (1999) 032 hep-th/9908142. 
[5] J. de Boer, P.A. Grassi and P. van Nieuwenhuizen, Non-commutative superspace from string theory, Phys. Lett. B 574 (2003) 98 hep-th/0302078.

[6] S. Ferrara, M.A. Lledó and O. Macia, Supersymmetry in noncommutative superspaces, JHEP 09 (2003) 068 hep-th/0307039.

[7] D. Klemm, S. Penati and L. Tamassia, Non(anti)commutative superspace, Class. and Quant. Grav. 20 (2003) 2905 hep-th/0104190.

[8] E. Ivanov, O. Lechtenfeld and B. Zupnik, Nilpotent deformations of $N=2$ superspace, JHEP 02 (2004) 012 hep-th/0308012.

[9] I. Martin and A. Restuccia, Symplectic connections, noncommutative Yang-Mills theory and supermembranes, Nucl. Phys. B 622 (2002) 240 hep-th/0108046.

[10] S.V. Ketov and S. Sasaki, Non-anticommutative $N=2$ supersymmetric $\mathrm{SU}(2)$ gauge theory, Phys. Lett. B 597 (2004) 105 hep-th/0405278.

[11] K. Ito and S. Sasaki, Non(anti)commutative $N=2$ supersymmetric gauge theory from superstrings in the graviphoton background, JHEP 11 (2006) 004 [hep-th/0608143].

[12] L. Cornalba and R. Schiappa, Nonassociative star product deformations for D-brane worldvolumes in curved backgrounds, Commun. Math. Phys. 225 (2002) 33 hep-th/0101219.

[13] T. Asakawa and I. Kishimoto, Noncommutative gauge theories from deformation quantization, Nucl. Phys. B 591 (2000) 611 hep-th/0002138.

[14] B.v. Fedosov, A Simple geometrical construction of deformation quantization, J. Diff. Geom. 40 (1994) 213.

[15] B. Fedosov, Deformation quantization and index theory, Akademie-Verl., Berlin Germany (1996), pg. 325.

[16] I. Kishimoto, Fuzzy sphere and hyperbolic space from deformation quantization, JHEP 03 (2001) 025 hep-th/0103018.

[17] F. Peña, Path formulation for noncommutative gauge theories, Tesis de Maestría, Univeridad Simón Bolívar, Venezuela (2007).

[18] M. Bordemann, H.-C. Herbig and S. Waldmann, BRST cohomology and phase space reduction in deformation quantisation, Commun. Math. Phys. 210 (2000) 107 math.QA/9901015.

[19] M. Bordemann, On the deformation quantization of superPoisson brackets, q-alg/9605038.

[20] M. Bordemann, The deformation quantization of certain super-Poisson brackets and BRST cohomology, prepared for Conference Moshe Flato, 5-8 September 1999, Dijon, France, math.DG/0003218.

[21] M. Kontsevich, Deformation quantization of Poisson manifolds, I, Lett. Math. Phys. 66 (2003) 157 q-alg/9709040.

[22] V.S. Varadarajan, Supersymmetry for mathematicians: an introduction, Courant Institute of Mathematical Sciences, New York U.S.A. (2004), pg. 300.

[23] P. Deligne et al., Quantum fields and strings: a course for mathematicians. Vol. 1 and vol. 2, American Mathematical Society, Providence U.S.A. (1999).

[24] P. Deligne and D.S. Freed, Supersolutions, hep-th/9901094. 
[25] J. Wess and J. Bagger, Supersymmetry and supergravity, Princeton University Press, Princeton U.S.A. (1992), pg. 259.

[26] F. Bayen, M. Flato, C. Fronsdal, A. Lichnerowicz and D. Sternheimer, Deformation theory and quantization. 2. Physical applications, Ann. Phys. (NY) 111 (1978) 111.

[27] M. Rothstein, The structure of supersymplectic manifolds, SISSA-43/90/FM.

[28] H.O.M. Khudaverdian, Semidensities on odd symplectic supermanifolds, Commun. Math. Phys. 247 (2004) 353.

[29] B. Kostant, Graded manifolds, graded Lie theory, and prequantization, in Bonn 1975 Proceedings, Differential geometrical methods in mathematical physics, Berlin 1977. 